Invited Editorial Focus

\title{
Novel Ligands for Platelet Glycoprotein VI
}

\author{
Meinrad Gawaz ${ }^{1}$ \\ ${ }^{1}$ Medizinische Klinik III, Eberhard Karls Universität Tübingen, \\ Tübingen, Germany
}

Thromb Haemost 2018;118:435-436.

Platelet hyperreactivity is a major risk factor for thromboischaemic events such as myocardial infarction and stroke. ${ }^{1}$ Antiplatelet therapy has substantially improved morbidity and mortality in patients with acute and chronic cardiovascular diseases. In patients with high risk for thromboischaemic events, dual-antiplatelet therapy (DAPT) consisting of acetyl salicylic acid and $\mathrm{P}_{2} \mathrm{Y}_{12}$ inhibitors has become the standard antithrombotic therapy for secondary prevention. ${ }^{2}$ However, current antiplatelet therapy (especially with DAPT) is associated with increased bleeding complications, which is associated with various clinical risk factors ${ }^{3,4}$ but also with enhanced mortality. ${ }^{5}$

Especially in patients with acute ischaemic stroke, secondary intracerebral bleeding is an imminent threat for patient recovery and disease progression, which may be further promoted by concomitant antiplatelet therapy. ${ }^{6}$ Thus, novel antithrombotic strategies are required with a favourable balance between thrombosis and bleeding.

In the past, the platelet collagen receptor glycoprotein $\mathrm{VI}$ (GPVI) has got considerable attention in controlling thrombosis. ${ }^{7}$ GPVI is a surface receptor primarily expressed in the megakaryocyte/platelet system. At the site of vascular injury, platelets adhere to exposed immobilized collagen within the subendothelium and become activated. Platelet interaction with fibrous collagen is a critical step of platelet-dependent thrombus formation. Adhesion of platelets with collagen is mediated both via GPVI and integrin $\alpha_{2} \beta_{1}$, and both receptors are constitutively expressed on the plasma membrane of platelets. ${ }^{8}$ GPVI is a 58 - to $62-\mathrm{kD}$ platelet transmembrane glycoprotein that belongs to the immunoglobulin family. GPVI consists of two extramembrane immunoglobulin domains, a transmembrane helix and a short cytosolic domain. GPVI exists in both monomeric and dimeric form on the platelet surface and interacts non-covalently with the Fc receptor $\gamma$-chain $(\mathrm{FcR} \gamma) .{ }^{9}$ Dimerization of GPVI is activation dependent and a prerequisite for high-affinity binding for collagen. GPVI monomers only weakly bind to immobilized collagen. Besides collagen, other ligands for GPVI have been described including fibronectin, vitronectin, laminin, adiponectin, EMMPRIN and fibrin. ${ }^{10} \mathrm{~A}$ profound understanding of GPVI binding to ligands other than collagen is a prerequisite for development of specific drugs targeting GPVI.

At the site of atherosclerotic plaque rupture, tissue factor (TF) is generated and induces fibrin formation and promotion of thrombus formation and consolidation. Recently, fibrin has been postulated as novel ligand for GPVI. ${ }^{11-13}$

In the current issue of Thrombosis and Haemostasis, Ebrahim et $\mathrm{al}^{14}$ challenge recent findings implying fibrin as novel ligand of GPVI. Ebrahim et al made use of fusion proteins consisting of the external domain of GPVI and the Fc part from IgG1 and IgG2, respectively. Both recombinant proteins are dimers of GPVI with high affinity for collagen. The authors tested binding of these recombinant GPVI molecules to fibrin derived from various sources (fibrin formed by thrombin-degraded purified fibrinogen or plasma or fibrin generated by human atherosclerotic carotid plaque specimen). Various receptor binding assays were performed. In none of the assays, specific binding of dimeric GPVI to fibrin fibrils could be detected. Furthermore, no specific binding activity of recombinant GPVI to fibrin could be observed in perfusion assays where fibrin is dynamically generated under the conditions of arterial blood flow. In contrast, in all these experiments, accumulation of GPVI on collagen fibres was always clearly visible. The authors made use of an elegant novel high-resolution imaging technology (structured illumination microscopy, SIM). With this imaging technique they were able to visualize fibrin formation in the environment of human atherosclerotic plaque-a situation closely related to the pathophysiology of vulnerable plaque rupture in humans. Again in none of these dynamic imaging experiments, binding of the GPVI receptor to fibrin could be documented.

In light of recent reports that postulate fibrin as a novel GPVI-ligand and the current data, ${ }^{11-13}$ the question arises whether fibrin is a ligand for GPVI? Previous studies made use of various genetic mouse models (GPVI deficiency or transgenic humanized GPVI mice) to validate the hypothesis 
of GPVI-fibrin interaction. Both mouse studies convincingly show that GPVI is involved in thrombin generation and clot formation which depended on fibrin polymerization. Furthermore, the pattern of platelet signaling induced by thrombin in the presence of fibrinogen and antagonist GPIIb-IIIa was similar to that induced by GPVI activation. Thus, the conclusion of both mouse studies that fibrin is a direct ligand of GPVI is primarily based on indirect measures of GPVI function. Although genetic deficiency of GPVI is a well-defined model, the results of these studied may also be modulated by so far undisclosed changes of platelet function due to genetic receptor deletion that might affect fibrinmediated platelet activation independent of direct interaction with GPVI. Mammadova-Bach et al also show significant direct binding activity of recombinant GPVI-Fc to fibrin in a fluorescence-based binding assay and Alshehri et al confirmed direct fibrin interaction of GPVI using a monomeric ectodomain of GPVI isolated from platelet-rich plasma. Thus, there are significant methodological differences between published studies and the present work of Ebrahim and colleagues in the current issue of Thrombosis and Haemostasis that might help explain the conflicting results. However, none of the previous published studies evaluated the interaction of GPVI with fibrin in an experimental system that is closely related to the pathophysiology of atherothrombosis. Ebrahim et al convincingly show that in the surrounding of human atherosclerotic tissue, the interaction of GPVI with fibrin is a prominent mechanism involved in atherothrombosis.

In recent years, GPVI has become an attractive target to modulate atherothrombosis. Previous studies have shown that inhibiting GPVI receptor function (by anti-GPVI antibodies) or competition for common collagen binding sites (by soluble dimeric GPVI receptor) effectively inhibits thrombosis. ${ }^{15,16}$ At least the strategy using the dimeric GPVI receptor to control atherothrombosis has little impact on bleeding, as it primarily controls thrombus formation at the site of vascular injury where collagen becomes exposed to the blood stream with limited systemic effects on haemostasis. ${ }^{17}$ Thus, lesion-directed inhibition of thrombosis via dimeric GPVI-Fc is an attractive pharmacological approach in patients with acute thrombo-ischaemic diseases such as myocardial infarction or ischemic stroke with limited risk for bleeding. At present, clinical phase II studies are currently performed to assess the feasibility and safety of dimeric GPVI-Fc in symptomatic coronary (NCT03312855) and carotid artery disease (NCT01645306).

Besides collagen, multiple other ligands have been described to bind to GPVI. However, in contrast to GPVIcollagen interaction, the functional consequences of these novel ligands for GPVI-dependent platelet function and thrombus formation are poorly understood. A deeper insight of GPVI-ligand interaction is necessary to define the future therapeutic role of GPVI inhibition.

\section{References}

1 Reny JL, Fontana P, Hochholzer W, et al. Vascular risk levels affect the predictive value of platelet reactivity for the occurrence of MACE in patients on clopidogrel. Systematic review and metaanalysis of individual patient data. Thromb Haemost 2016;115 (04):844-855

2 Elmariah S, Mauri L, Doros G, et al. Extended duration dual antiplatelet therapy and mortality: a systematic review and meta-analysis. Lancet 2015;385(9970):792-798

3 Capodanno D. Risk stratification with the DAPT score: carefully read the instructions and use as intended. Thromb Haemost 2017;117(10):1836-1839

4 Harada Y, Michel J, Lohaus R, et al. Validation of the DAPT score in patients randomized to 6 or 12 months clopidogrel after predominantly second-generation drug-eluting stents. Thromb Haemost 2017;117(10):1989-1999

5 Kwok CS, Rao SV, Myint PK, et al. Major bleeding after percutaneous coronary intervention and risk of subsequent mortality: a systematic review and meta-analysis. Open Heart 2014;1(01): e000021

6 Paciaroni M, Agnelli G, Ageno W, Caso V. Timing of anticoagulation therapy in patients with acute ischaemic stroke and atrial fibrillation. Thromb Haemost 2016;116(03):410-416

7 Varga-Szabo D, Pleines I, Nieswandt B. Cell adhesion mechanisms in platelets. Arterioscler Thromb Vasc Biol 2008;28(03):403-412

8 Gawaz M, Vogel S, Pfannenberg C, Pichler B, Langer H, Bigalke B. Implications of glycoprotein VI for theranostics. Thromb Haemost 2014; 112(01):26-31

9 Moroi M, Jung SM. Platelet glycoprotein VI: its structure and function. Thromb Res 2004;114(04):221-233

10 Chatterjee M, Gawaz M. Clinical significance of receptor shedding-platelet GPVI as an emerging diagnostic and therapeutic tool. Platelets 2017;28(04):362-371

11 Alshehri OM, Hughes CE, Montague S, et al. Fibrin activates GPVI in human and mouse platelets. Blood 2015;126(13):1601-1608

12 Mammadova-Bach E, Ollivier V, Loyau S, et al. Platelet glycoprotein VI binds to polymerized fibrin and promotes thrombin generation. Blood 2015;126(05):683-691

13 Induruwa I, Moroi M, Bonna A, et al. Platelet collagen receptor Glycoprotein VI-dimer recognizes fibrinogen and fibrin through their D-domains, contributing to platelet adhesion and activation during thrombus formation. J Thromb Haemost 2017. Doi: $10.1111 /$ jth.13919

14 Ebrahim M,Jamasbi J, Adler K, et al. Dimeric glycoprotein VI binds to collagen but not to fibrin. Thromb Haemost 2018;118(02):351-361

15 Massberg S, Gawaz M, Grüner S, et al. A crucial role of glycoprotein VI for platelet recruitment to the injured arterial wall in vivo.J Exp Med 2003;197(01):41-49

16 Massberg S, Konrad I, Bültmann A, et al. Soluble glycoprotein VI dimer inhibits platelet adhesion and aggregation to the injured vessel wall in vivo. FASEB J 2004;18(02):397-399

17 Ungerer M, Rosport K, Bültmann A, et al. Novel antiplatelet drug revacept (Dimeric Glycoprotein VI-Fc) specifically and efficiently inhibited collagen-induced platelet aggregation without affecting general hemostasis in humans. Circulation 2011;123(17):1891-1899 